\title{
THE PASSAGE OF PHOTOELECTRONS THROUGH METALS.
}

\author{
By K. T. Compton and L. W. Ross.
}

\begin{abstract}
Synopsis.-Theoretical expressions are derived giving the rate of escape of photo-electrons from the surface of a thin metallic film as a function of its thickness. These expressions result from various assumptions regarding the probability of escape of an electron photoelectrically excited at a given depth within the metal.

The photoelectric currents from films of platinum and of gold of various thicknesses are measured for different wave-lengths of light, and the results are found to be consistent with the view that the average distance in any direction which an excited electron may move without losing its ability to escape is about 2.67 (10 $)^{-7} \mathrm{~cm}$. in platinum and $5.0(\mathrm{ro})^{-7} \mathrm{~cm}$. in gold, and that the probability of going a given distance without losing its ability to escape falls off exponentially with the distance. This average distance is shown to be independent of the initial kinetic energy of the electron. There is evidence that platinum is first deposited in a relatively unstable and electropositive state, and that it spontaneously changes into the ordinary stable form.

A critical discussion and comparison of methods of measuring film thicknesses shows that they may be relied upon if proper precautions are taken. Various interesting properties of these thin films are illustrated and discussed.
\end{abstract}

INTRODUCTION.-Ladenburg ${ }^{1}$ was the first to prove that electrons, excited photoelectrically within a metal by ultra-violet light, may travel some distance through the metal before escaping from its surface. $\mathrm{He}$ deposited nickel electrolytically on glass, and showed that the photoelectric currents from the films increased with increasing film thickness up to about $2(\mathrm{ro})^{-4} \mathrm{~cm}$. This result was qualitatively confirmed by Rubens and Ladenburg, ${ }^{2}$ who showed that the ratio of the photoelectric currents on the incident and emergent sides of a piece of gold foil was about $100: I$, while the ratio of the incident and emergent light intensities was about $\mathrm{I}, 000: \mathrm{I}$.

In I9I3, Partzsch and Hallwachs ${ }^{3}$ published a criticism of the quantitative results of Ladenburg's measurements, and also made a theoretical and experimental study of the optical and photoelectric properties of thin metal films deposited on quartz. The theoretical treatment in the present paper is based on, and is an extension of, the work of Partzsch and Hallwachs.

The experimental measurements here recorded are in many respects

${ }_{1}$ Ann. d. Phys., I2, p. 558, 1903.

2 Ber. d. D. Phys. Ges., p. 749, I907.

${ }^{3}$ Ann. d. Phys., 4I, p. 247, I9I3. 
similar to measurements published by Robinson, ${ }^{1}$ and in the main confirm his results. Certain refinements had to be added to his experimental procedure, however, in order to attain sufficient accuracy to permit a quantitative application of the theory.

METHOD.-The general plan of the experiment was to allow ultraviolet light of definite wave-length to enter a metal film, deposited on a quartz plate, from the quartz side and to measure the total photoelectric emission from the film. This was done with films of various thicknesses, maintaining other conditions constant, so that the photoelectric emission was obtained as a function of the film thickness. The nature of the variation of emission with thickness depends on the distance rate of absorption of light in the film and on the probability that an electron, photoelectrically excited by the light at any point in the film, may reach and escape from the surface of the film. The rate of absorption of light in the film is known, or may easily be measured. Thus the experimental results depend ultimately on the probability of the escape of an electron from any point in the film. Various assumptions regarding the form of the expression of this probability, associated with various assumptions regarding the passage of electrons through the metal, may

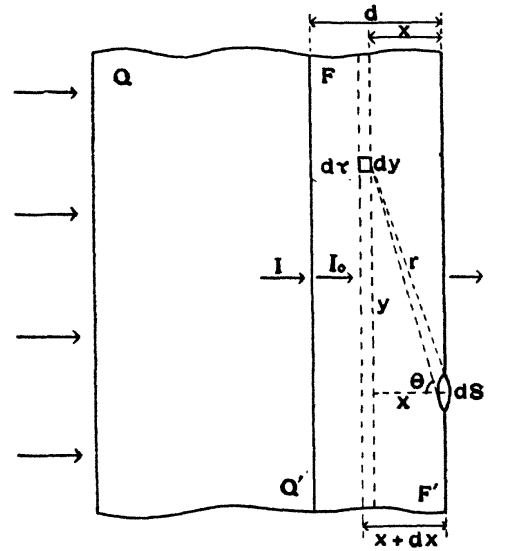

Fig. 1. therefore be tested by the experimental results. In the following section several such assumptions are put into forms suitable for quantitative tests.

TheORY.-Let $Q Q^{\prime}$ and $F F^{\prime}$ in Fig. I represent sections of the quartz plate and metal film, respectively, the thickness of the latter being $d$. Light of intensity $I$ strikes the film from the quartz side, but part of it is reflected, so that $I_{0}$ represents the intensity of the light which enters the film. Since the reflecting power of the film varies with its thickness, $I_{0}$ is a function of $d$.

Let $d x$ be the thickness of a layer of the film distant $x$ from its free surface. The intensity of the light in this layer is $I_{0} \epsilon^{-a(d-x)}$, where $\alpha$ is the coefficient of absorption of the light. If we let $\nu$ represent the number of electrons which are photoelectrically excited per unit time, per unit volume, per unit light intensity, then the rate at which electrons

${ }^{1}$ Phil. Mag., 25, p. 115, 1913; ibid., 32, p. 421, 1916. 
are excited in the layer $d x$ is

$$
\nu A I_{0} \epsilon^{-a(d-x)} d x,
$$

where $A$ is the area of the film. From symmetry it is evident that half of these start moving toward the free surface. ${ }^{1}$

Let $F(x)$ be the probability that an electron will move a distance whose component normal to the surface is $x$ without losing its ability to escape. Then the rate of escape from the film of electrons which start from the layer $d x$ is

$$
d N=\frac{\nu A}{2} \underline{I}_{0} \epsilon^{-a(d-x)} F(x) d x .
$$

The total rate of emission of electrons is therefore

$$
N=\frac{\nu A I_{0}}{2} \epsilon^{-\alpha d} \int_{0}^{d} \epsilon^{\alpha x} F(x) d x .
$$

SPECIAl Cases.-(a) Assume that the number of electrons which retain ability to escape falls off exponentially with distance moved normally to the surface, or

$$
F(\dot{x})=\epsilon^{-\beta x},
$$

where $I / \beta$ is the average distance which an electron can move normally to the surface without losing its ability to escape. Then the integration in equation ( $\mathrm{I}$ ) is easily performed, giving

$$
N=\frac{\nu A I_{0}}{2} \frac{\epsilon^{-a d}-\epsilon^{-\beta d}}{\beta-\alpha}
$$

which is identical with the equation derived by Partzsch and Hallwachs. ${ }^{2}$

(b) Assume that the number of electrons which retain ability to escape falls off exponentially with the distance moved (in any direction).-Consider those electrons which start toward the surface from an element of volume $d \tau$ in the layer $d x$ and escape from an element of the free surface $d S$. Of these, the fraction $d S \cos \theta / 2 \pi r^{2}$ start toward $d S$; but of these, only the fraction $\epsilon^{-\gamma^{r}}$ succeed in escaping, according to our present assumptions. Thus

$$
\frac{d S \cos \theta}{2 \pi r^{2}} \epsilon^{-\gamma^{r}}
$$

1 This assumes that the electrons begin to move with equal probabilities of motion in all directions and therefore in directions which are independent of the orientation of the electric vector in the light wave. There is possibly some experimental evidence that this assumption is not strictly true. If the electron is ejected from the atom by a direct pull from the electric field of the light wave the present assumption is probably seriously in error. If, however, the photoelectric emission is a secondary effect caused by a storing up of energy in the atom and its consequent instability, the assumption is valid.

2 Loc. cit. 
is the probability that an electron, starting toward the surface from $d \tau$, will escape from $d S$. It is to be noted that this probability is the same for electrons starting from any point in $d x$ at a distance between $y$ and $y+d y$ from $O$. Thus the probability that an electron, starting toward the surface from any point in the layer $d x$, will escape from $d S$ is

$$
\frac{\mathrm{I}}{A} \int_{y=0}^{y=\infty} \frac{d S \cos \theta}{2 \pi r^{2}} \epsilon^{-\gamma^{r}} \cdot 2 \pi y d y,
$$

since the layer may be considered to extend to infinity in comparison with the depth $x$. Finally, the probability that an electron, starting toward the surface from $d x$, will escape, is found by substituting $A$ for $d S$, and is

$$
F(x)=\int_{0}^{\infty} \frac{\cos \theta}{r^{2}} y \epsilon^{-\gamma^{r}} d y
$$

This expression may be written

$$
F(x)=x \int_{x}^{\infty} \frac{\mathbf{I}}{r^{2}} \epsilon^{-\gamma^{r}} d r
$$

of which the solution is

$$
F(x)=\epsilon^{-\gamma^{x}}+\gamma x E i(-\gamma x),
$$

where $E i(-\gamma x)$ represents the exponential integral

$$
E i(-\gamma x)=-\int_{x}^{\infty} \frac{\mathbf{I}}{r} \epsilon^{-\gamma r} d r
$$

whose value for various values of $(\gamma x)$ is given in Laska's "Sammlung von Formeln."

On this assumption, therefore, the total rate of electron emission from the film should be, by equation (I),

$$
N=\frac{\nu A I_{0}}{2} \epsilon^{-\alpha d} \int_{0}^{d} \epsilon^{\alpha x}\left[\epsilon^{-\gamma x}+\gamma x E i(-\gamma x)\right] d x .
$$

The integral of this expression may be easily evaluated by graphical methods. ${ }^{1}$

1 This equation may be integrated by parts after substituting for $E i(-r x)$ its alternative form given above. In performing this integration it is necessary to express the limits of integration of one of the terms by

$$
\begin{aligned}
& \int_{0}^{d} f(x) d x=\int_{0}^{\infty} f(x) d x-\int^{\infty} f(x) d x \\
& \text { and to remember that } \quad \int_{0}^{\infty} \frac{\epsilon^{-r x}-\epsilon^{-(r-\alpha) x}}{x} d x=\log \frac{r-\alpha}{r} . \\
& \text { We thus find for the solution } \\
& \begin{array}{r}
N=\frac{\nu 1_{0} A}{2}\left\{\frac{\mathrm{I}}{\alpha}\left(\epsilon^{-r d}-\epsilon^{-\alpha d}\right)+r\left(\frac{d}{\alpha}-\frac{\mathrm{I}}{\alpha^{2}}\right) E i(-r d)\right. \\
\left.+\frac{r}{\alpha^{2}} \epsilon^{-\alpha d} E i(-(r-\alpha) d)+\frac{r}{\alpha^{2}} \epsilon^{-\alpha d} \log \frac{r}{r-\alpha}\right\} .
\end{array}
\end{aligned}
$$


In this expression, $\mathrm{I} / \gamma$ represents the average distance in any direction which an electron may move without losing its ability to escape. ${ }^{1}$

(c) Assume that an electron loses energy in proportion to the distance moved through the metal.-Let $\sigma$ be the energy lost per centimeter path. The electron starts with an amount of energy $h \nu$, where $h$ is Planck's constant and $\nu$ is the frequency of the exciting light; of this energy, an amount $h \nu_{0}$ is necessary to enable it to escape from the surface of the metal. Therefore

$$
\sigma r_{0}=h\left(\nu-\nu_{0}\right)
$$

determines the greatest distance $r_{0}$ which an electron can move in the metal without losing its ability to escape. As in case $(b)$, the fraction $d S \cos \theta / 2 \pi r^{2}$ of the electrons starting toward the free surface from $d \tau$ move toward $d S$. All these will escape if $r<r_{0}$, and none if $r>r_{0}$. Thus the probability that an electron starting toward the surface from the layer $d x$ will escape from $d S$ is

$$
\frac{\mathrm{I}}{A} \int_{0}^{\sqrt{r_{0}^{2}-x^{2}}} \frac{d S \cos \theta}{2 \pi r^{2}} \cdot 2 \pi y d y=\frac{d S}{A}\left(\mathrm{I}-\frac{x}{r_{0}}\right)
$$

if $x<r_{0}$ and is zero if $x>r_{0}$. Therefore

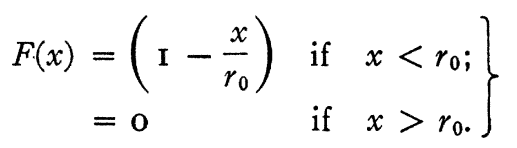

By equation (I), if $d>r_{0}$,

$$
\begin{aligned}
N & =\frac{\nu A I_{0}}{2} \epsilon^{-\alpha d} \int_{0}^{r_{0}}\left(\mathrm{I}-\frac{x}{r_{0}}\right) \epsilon^{a x} d x \\
& =\frac{\nu A I_{0}}{2} \epsilon^{-\alpha d}\left[-\frac{\mathrm{I}}{\alpha^{2} r_{0}}\left(\epsilon^{a r_{0}}-\mathrm{I}\right)-\frac{\mathrm{I}}{\alpha}\right],
\end{aligned}
$$

Although this equation permits numerical computations to be made, such computations are neither as rapid nor as accurate as those made by a graphical solution of equation (5). The inaccuracy is due to the fact that the value for $N$ is expressed as the difference between relatively large terms, and the tables of $E i(-r x)$ are not sufficiently accurate to give the difference with high percentage accuracy.

1 We have implicitly assumed that the "ability to escape" depends on the velocity but not on the direction of motion of an electron at the surface. This is objectionable, since the chance of escaping against the attraction of the induced positive charge left on the film is certainly dependent on direction as well as speed of motion of an electron. The mechanism of "escape" and the nature of the work done in escaping is so little understood, however, that we have neglected this factor. If most of the work done in escaping is done within distances from the surface which are of atomic order of magnitude, the present assumption will probably not be seriously in error. It can furthermore be shown that the relations between $N, \alpha$ and $r$ are such as to reduce any error from this cause to a minimum. 
and if $d<r_{0}$

$$
\begin{aligned}
N & =\frac{\nu A I_{0}}{2} \epsilon^{-\alpha d} \int_{0}^{d}\left(\mathrm{I}-\frac{x}{r_{0}}\right) \epsilon^{a x} d x \\
& =\frac{\nu A I_{0}}{2}\left[\left(\mathrm{I}-\epsilon^{-\alpha d}\right)\left(\frac{\mathrm{I}}{\alpha}+\frac{\mathrm{I}}{\alpha^{2} r_{0}}\right)-\frac{d}{\alpha r_{0}}\right] .
\end{aligned}
$$

Thickness of Film giving Maximum Photoelectric Emission.-It is much easier, in practice, to determine or test values of $\beta, \gamma$ and $\sigma$ by finding the thickness of film giving maximum emission than by attempting to fit the entire graphs of the theoretical equations to the experimental curves. We shall therefore discuss the conditions under which maximum emission is obtained in the three cases considered above.

(a) By equating to zero the derivative of equation (3) with respect to film thickness $d$ we find the thickness $d_{m}$ for maximum emission to be given by

$$
d_{m}=\frac{\mathrm{I}}{\beta-\alpha} \log \frac{\beta-\left(\frac{\mathrm{I}}{I_{0}} \frac{\partial I_{0}}{\partial d}\right)_{m}}{\alpha-\left(\frac{\mathrm{I}}{I_{0}} \frac{\partial I_{0}}{\partial d}\right)_{m}},
$$

in which the term $\mathrm{I} / I_{0} \partial I_{0} / \partial d$ arises from the variation of the reflecting power of the quartz-film surface with thickness and may be determined experimentally, as described later. Taking experimental values of this term and of the coefficient of light absorption $\alpha$ we find the values of $d_{m}$ for various values of $\alpha$ and $\beta$ shown graphically in Fig. 2. If $d_{m}$ and $\alpha$

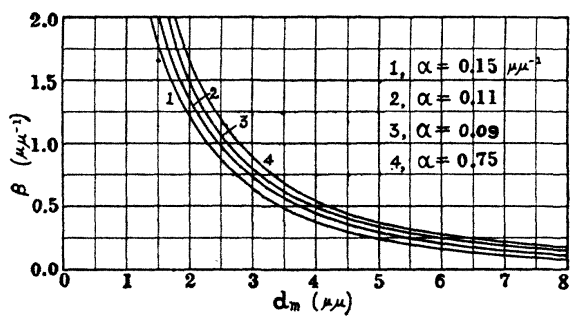

Fig. 2.

are determined experimentally, the appropriate value of $\beta$ may be found from these curves.

(b) To find the maximum value of $N^{N}$ from equation (5) the easiest method is to actually plot the equation, as is done in Fig. 3 for various values of $\gamma^{\mathrm{i}}$ From these and similar curves the values of $d_{m}$ appropriate

I In Fig. 3 the curves for various values of $r$ are drawn so as to merge into the curve giving the percentage of transmitted light, thus calling attention to the fact that for thick films the photoelectric emission varies with thickness in practically the same manner as does the transmitted light. To do this, however, the four curves had to be plotted to four different scales. If drawn to the same scale the maximum values of $N$ for the four curves $\mathrm{I}, 2,3$ and 4 would be $21.3,39.9,70.9$ and 121.7 respectively. 
to various values of $\gamma$ and $\alpha$ have been determined and plotted in Fig. 4, whence $\gamma$ may be found if $\alpha$ and $d_{m}$ are experimentally determined.

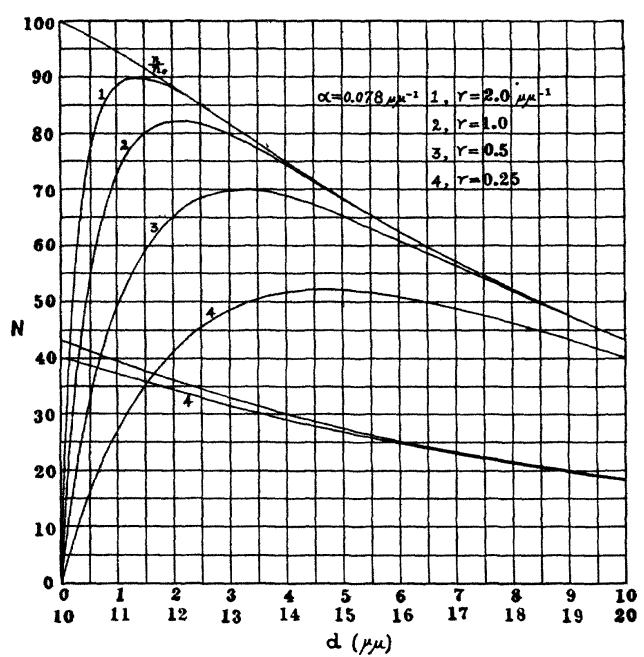

Fig. 3.

(c) In case energy is lost in proportion to the distance moved through the metal, the thickness for maximum emission is given by differentiation of equation (9), whence

$$
d_{m}=\frac{\mathbf{I}}{\alpha} \log \left(\mathrm{I}+\alpha r_{0}\right),
$$

where $r_{0}$ is defined by equation (6) as the greatest distance which an electron can go through the metal without losing its ability to escape.

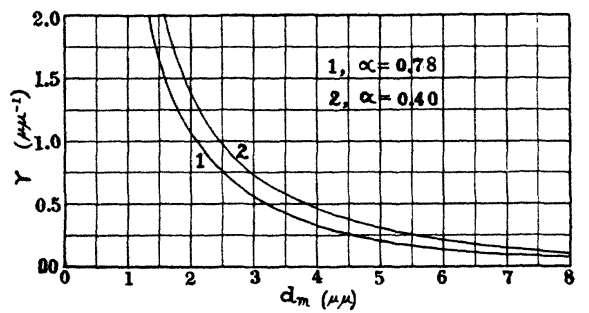

Fig. 4.

Since $\alpha d_{m}$ is an extremely small quantity, equation (I I) may be written without appreciable error, $\alpha d_{m}=\alpha r_{0}$, whence, by equation (6)

$$
d_{m}=\frac{h\left(\nu-\nu_{0}\right)}{\sigma} .
$$




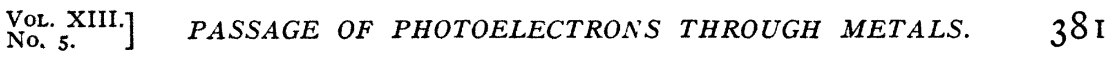

If this were the actual type of energy loss, the thickness of film giving maximum current should be proportional to $\left(\nu-\nu_{0}\right)$, which is the difference between the frequency of the exciting light and the least frequency of light which will produce photoelectric emission.

Apparatus.- In order to test the foregoing assumptions and to measure some of the quantities involved, metallic films were deposited on quartz plates by the method of "sputtering," in a vessel whose essential features are shown by Fig. 5. Transparent quartz plates one centimeter square and one millimeter thick were carefully cleaned in boiling caustic potash, nitric acid and distilled water and mounted by a touch of sealing wax over holes in a brass disk, as at $P$. Twelve such plates were mounted on the disk, which was then placed in the vacuum chamber shown in Fig. 5, so that the plates could be placed in turn under the cathode $C$ by turning the ground glass joint $J$. A stationary brass shield $S S^{\prime}$ was held just above the disk $N N^{\prime}$ so as to shield from the discharge all the plates except the one $P$ below the cathode. The vessel was rendered air tight by melting wax in the groove $G G^{\prime}$. Great care was taken to keep the inside of the vessel free from wax or grease, and where wax was necessary it was entirely shielded from the discharge. The exhaust tube was connected through a

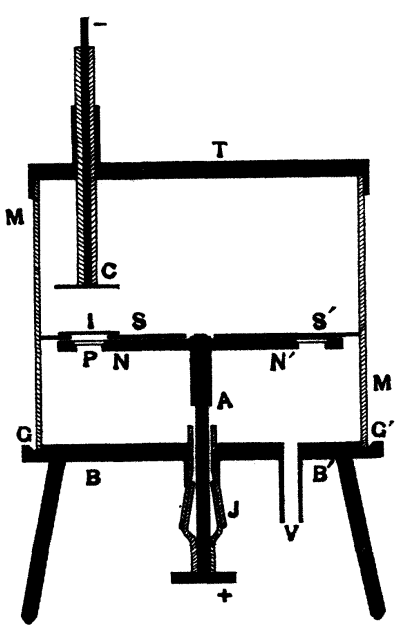

Fig. 5. liquid air trap to a McLeod gauge and Gaede pump. The source of the discharge current was a Snook high potential transformer designed to give a very steady discharge.

Measurement of Thickness of Films.-Three methods were employed to measure the thickness of the films. Of these, the most direct method was to weigh the thickest film, thence calculate its thickness and take the thickness of the other films proportional to their times of exposure to "sputtering." B. Pogány ${ }^{1}$ has shown that this method is accurate if the sputtering conditions are maintained constant. In order to have constant conditions we adopted the following procedure. An iron disk $I$ was placed over the hole in the shield $S S^{\prime}$ so that none of the quartz plates was exposed to the discharge. The discharge in the vessel was then continued for about two hours, until no more gas could be detected coming from the walls of the vessel under the influence of the discharge.

1 Phys. Zeit., I5, p. 685, I9I4. 
Then the iron disk $I$ was removed by a magnet and the plates were exposed successively to the discharge. They were exposed in the following order: $\mathrm{I}, 2,3, \cdots, \mathrm{II}, \mathrm{I} 2, \mathrm{I} 2, \mathrm{II}, \cdots, 2, \mathrm{I}, \mathrm{I}, 2, \cdots, \mathrm{I} 2, \mathrm{I} 2, \cdots, \mathrm{I}$; so that the effect of any change in sputtering conditions would be evenly distributed among the plates. During the entire process the gas pressure was maintained constant by a regulating valve. Ordinarily, the times of deposit ranged from I minute to Ioo or more minutes.

The second method employed involved a measurement of the absorption of light in the films. The disk $N N^{\prime}$ was mounted on an optical bench so that light from a mercury arc could be focused on any one of the films. The reflected or transmitted light was focused on the slit of a Hilger monochromatic illuminator provided with a linear thermopile, which served to measure the intensity of the light. By thus determining the intensity of the incident light, the reflection and absorption by the quartz plate and the intensities of the lights transmitted and reflected by the film covered plates, we found the fraction of the light entering the film which was absorbed by it. The coefficient of absorption of light of the wave-lengths used, $576.9 \mu \mu$ and 579.I $\mu \mu$, is accurately known, whence the thickness of each film could be calculated.

The third method involved the use of data concerning the variation of reflection of ultra-violet light with thickness of platinum films on quartz, published by Partzsch and Hallwachs. ${ }^{1}$ When light strikes a platinum film from the quartz side, they found that the intensity $I_{0}$ of light entering the film is less than the intensity $I$ of light incident on the quartz by an amount shown by curve I, Fig. 6 , for films of various thicknesses $d$. Ninety-two per cent. of the incident light is transmitted by a blank plate. From this we find that the ratio of the intensity of light transmitted by a blank plate to that transmitted by a plate with film of thickness $d$ is given by

$$
\frac{n}{n_{0}}=\frac{I_{0}}{.92} \epsilon^{-a d},
$$

in which $\alpha$ is known with considerable accuracy for the wave-length $253.6 \mu \mu$ as a result of a slight extra-polation of measurements made by Meier. ${ }^{2}$ Taking $\alpha=.078 \mu \mu^{-1}$, we obtain curve 2, Fig. 6. The intensity of the light transmitted by any film was taken to be proportional to the photoelectric current produced when this light fell upon a plate $P$ (Fig. 7) which could be placed in the path of the light. The ratio of this current to that produced by light which has passed through a blank plate was therefore equal to $n / n_{0}$. Thus the thickness of the film $d$ could be

1 Loc. cit.

2 Ann. d. Phys., 31, p. I0I 7, 1909. 
Vol. XIII.] PASSAGE OF PHOTOELECTRONS THROUGH METALS.
No. 5.

found directly from curve 2 , Fig. 6 , as soon as the ratio $n / n_{0}$ was determined.

The reliability of these methods of estimating film thickness is proven by a comparison of their results when applied to the same set of films.

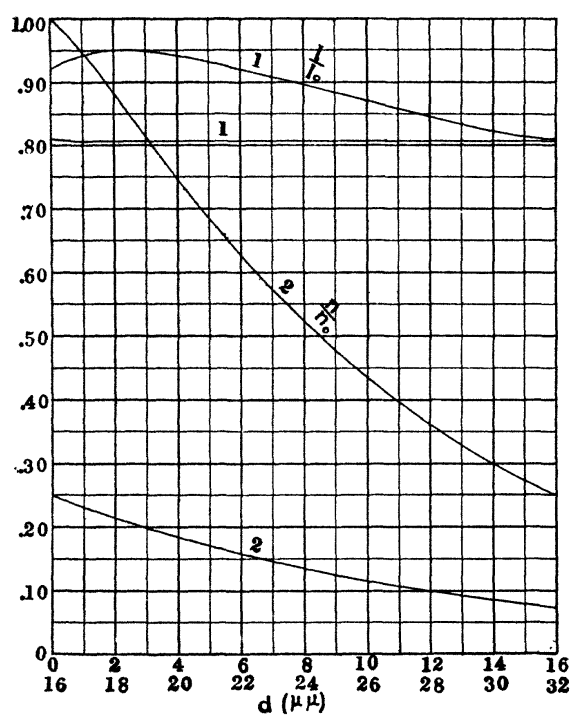

Fig. 6.

In Table I., $t$ represents the time of sputtering and $d_{1}, d_{2}, d_{3}$ are the thicknesses calculated by the three methods, respectively. The area

TABLE I.

\begin{tabular}{c|c|c|c|c}
\hline \hline No. & $t$ (sec.). & $d_{1}(\mu \mu)$. & $d_{2}(\mu \mu)$. & $d_{3}(\mu \mu)$. \\
\hline 1 & 0 & 0 & 0 & 0 \\
2 & 20 & 1.5 & 1.8 & 1.7 \\
3 & 30 & 2.3 & 2.5 & 2.5 \\
4 & 40 & 3.1 & 3.0 & 3.0 \\
5 & 50 & 3.8 & 3.8 & 3.9 \\
6 & 60 & 4.5 & 4.4 & 4.6 \\
7 & 70 & 5.3 & 5.5 & 5.5 \\
8 & 80 & 6.0 & 6.5 & 6.2 \\
9 & 90 & 6.8 & 6.8 & 6.6 \\
10 & 120 & 9.0 & 8.6 & 8.6 \\
11 & 150 & 11.3 & 11.8 & 11.6 \\
12 & 6000 & 452.0 & $?$ & $?$ \\
\hline
\end{tabular}

of plate no. 12 was $0.95 \mathrm{sq} . \mathrm{cm}$. and the mass of film deposited on it was $0.000925 \mathrm{gm}$. This film was so opaque that no estimates of its thickness could be made by the second and third methods. The experimental 
data which were used, with Curve 2, Fig. 6 , to find the values of $d_{3}$ are found in Table II.

Of these methods, the third was most convenient, and was used for most of the work with platinum films. The data for its use in dealing with gold films are lacking, so that we employed the second method in this case.

Photoelectric Measurements.-The photoelectric properties of these films were tested in the brass vessel shown in Fig. 7. Electrical contact between the films and the brass disk $N N^{\prime}$ was made by gold leaf, cemented with a little India ink. The disk was mounted on a shaft $A$ in such a

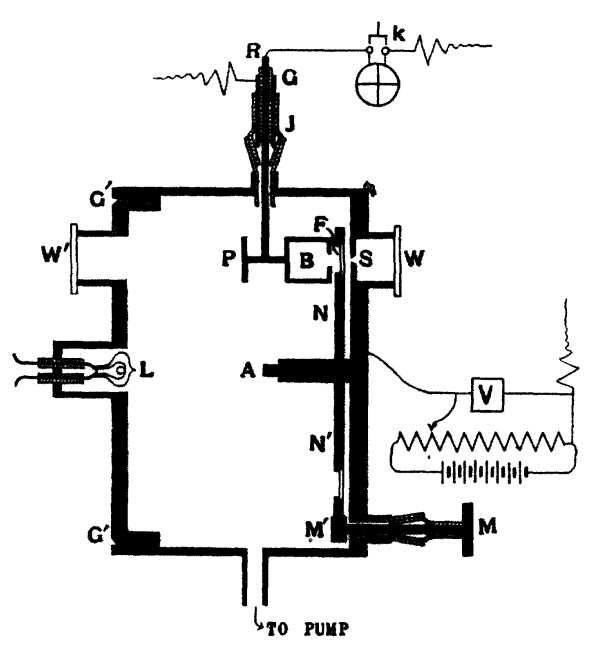

Fig. 7. manner that any film $F$ could be placed in the path of ultraviolet light from a quartz mercury arc and a $\mathrm{Hilger}$ monochromatic illuminator. This light entered through a quartz window $W$, was brought to a focus at the slit $S$, passed through the quartz plate and film and entered the hollow brass box $B$. This box was designed to reduce the amount of light returning to the film after reflection from the box, and a calculation proved that less than one per cent. of the photoelectric current from the film could be due to light reflected from the box.

The photoelectric current $N$ from the film $F$ passed to the box $B$ which served as the receiving electrode, and was connected with an electrometer which was used at a sensitiveness between 2,000 and 50,000 $\mathrm{mm}$. per volt, depending on the type of measurement being made. The vessel and films were charged to a negative potential of about io volts, indicated by the voltmeter $V$, and the insulation of the electrometer system was protected by the guard ring $G$.

In order to measure the intensity of the light transmitted by the film, the platinum plate $P$ was turned to the position of the box $B$ and the potential of the vessel changed to + ro volts, so that the electrometer measured the photoelectric current $n$ from the plate.

To facilitate quick substitution of one film for another, a second toothed wheel $M^{\prime}$, which could be rotated by the outside handle $M$, was made to engage the toothed disk $N N^{\prime}$. A small lamp $L$ illuminated the 
inside of the vessel, and the adjustment of any film to the position $F$ was visible when looking through the window $W^{\prime}$.

Experimental Results. Platinum.-Ten sets of platinum films were tested, each set being examined to determine $(a)$ the thickness of film giving maximum photoelectric emission, $(b)$ the effect of using different wave-lengths of exciting light and $(c)$ the nature of changes which occur in the films after they are deposited on the quartz plates. These points will be taken up separately.

(a) The Thickness of Film Giving Maximum Photoelectric Emission.Table II. contains typical experimental measurements. The numbers

TABLE II.

\begin{tabular}{c|c|c|c|c|c|c|c}
\hline No. & $N_{1}$. & $N_{2 .}$ & $N$. & $n_{1 .}$ & $n_{2 \cdot}$ & $n$. & $d(\mu \mu)$. \\
\cline { 2 - 8 } 1 & 0 & 0 & 0 & 191 & 186 & 188 & 0.0 \\
2 & 47 & 45 & 46 & 170 & 168 & 169 & 1.7 \\
3 & 74 & 68 & 71 & 162 & 158 & 160 & 2.5 \\
4 & 83 & 82 & 82 & 153 & 152 & 152 & 3.0 \\
5 & 81 & 81 & 81 & 145 & 139 & 142 & 3.9 \\
6 & 77 & 72 & 75 & 134 & 131 & 133 & 4.6 \\
7 & 67 & 68 & 67 & 122 & 124 & 123 & 5.5 \\
8 & 65 & 62 & 63 & 117 & 113 & 115 & 6.2 \\
9 & 67 & 67 & 67 & 112 & 112 & 112 & 6.6 \\
10 & 58 & 60 & 59 & 92 & 93 & 93 & 8.6 \\
11 & 31 & 29 & 30 & 71 & 71 & 71 & 11.6 \\
12 & 0 & 1 & 1 & 0 & 0 & 0 & $?$ \\
\hline
\end{tabular}

in the columns marked $N$ refer to the electrometer deflections per half minute due to the photoelectric currents from the films. Those marked $n$ refer to currents from the plate $P$ and measure the intensity of the light transmitted by the films; these data are used with Fig. 6 to determine the film thickness $d$. In order to avoid error due to "time" changes, such as photoelectric fatigue, all measurements were taken in order I, 2, 3, $\cdots, 1 \mathrm{I}, \mathrm{I} 2$ and back again $12, \mathrm{II}, \cdots, 2, \mathrm{I}$, as shown in the columns marked with subscripts I and 2. The means of these measurements are given in columns $N$ and $n$.

These results are shown graphically in Fig. $8(\mathrm{c})$. It is seen that there are two maxima, instead of the one predicted by the theory. It might be thought that this is due to some erratic photoelectric condition of films 9 and Io, if it were not for the fact that they were found in every one of the ten sets of platinum films tested. Other examples are shown in curves $(a),(b)$ and $(d)$, in which the ordinates have been changed by constant factors in order to avoid confusion in plotting. It appears that there is one maximum at $d=3.75 \mu \mu \pm .25 \mu \mu$ and another in the neighborhood of $d=7.5 \mu \mu \pm$ I.O $\mu \mu$. 
Numerous tests were made in an attempt to discover the cause of the second maximum. For one set of films mercury vapor was allowed free access to the sputtering and testing vessels, while in other tests all parts were carefully cleaned and mercury vapor excluded by liquid air traps, but no difference was observed. Apparently it made no difference

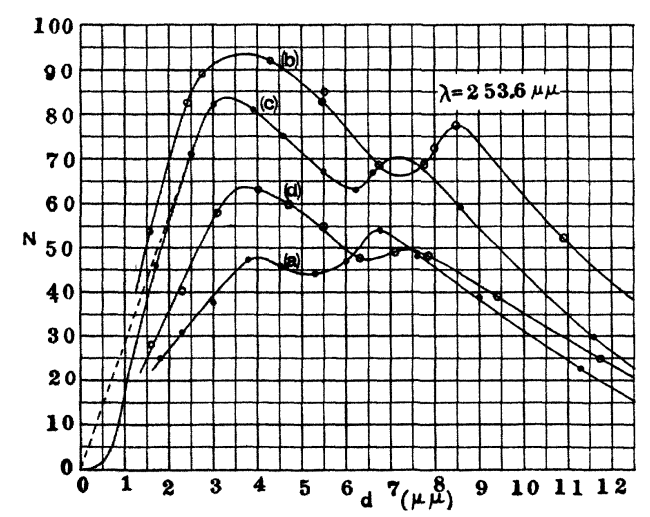

Fig. 8.

whether the films were allowed to stand in air or in vacuo. We have concluded that both maxima are really due to properties of the platinum films. The interpretation of these maxima is suggested by experiments discussed in the following sections.

One other feature of these curves is of interest. All the curves near the origin show an inflection, as if the photoelectric currents increased with thickness of film, first slowly and then more rapidly. If the quantity $\nu$ in our theoretical equations were constant, this portion of the curve would be straight, indicating proportionality between emission and thickness. Robinson, ${ }^{1}$ who has noticed this phenomenon, has supposed that it indicates less intrinsic photoelectric sensitiveness for thin than for thicker films. We have been able to prove, however, that $\nu$ really is constant, and that the inflection in the curves is due to a secondary cause, viz., lack of continuity of very thin films. When a very thin film is first exposed to the ultra-violet light, the rate of electrometer deflection falls off rapidly as the exposure is continued. If the light is shut off the film regains its original apparent sensitiveness only after a lapse of some minutes. If the initial rate of deflection instead of the total deflection in a relatively long time interval is used to determine $N$, it is found that the values of $N$ tend to lie on the theoretical straight line, such as the dotted line in curve $(c)$. This is much more marked

${ }^{1}$ Loc. cit. 
in the case of gold films than in the case of platinum films-which is in line with the observation that a greater thickness of gold than of platinum is required to give an electrically conducting layer. ${ }^{1}$ The upward concavity of the curves near the origin is apparently to be explained, therefore, by the effect of isolated regions of the film which quickly charge up to such positive potentials as to reduce the rate of emission from these and contiguous parts of the film. This phenomenon is easily detected by the failure to get electrometer deflections which are proportional to time, but it was never observed in the case of a platinum film thicker than $2 \mu \mu$.

(b) The Effect of Using Different Wave-lengths of Exciting Light.-The influence of the wave-length of exciting light upon the character of the emission is shown by the curves of Fig. 9. All show the double maximum,

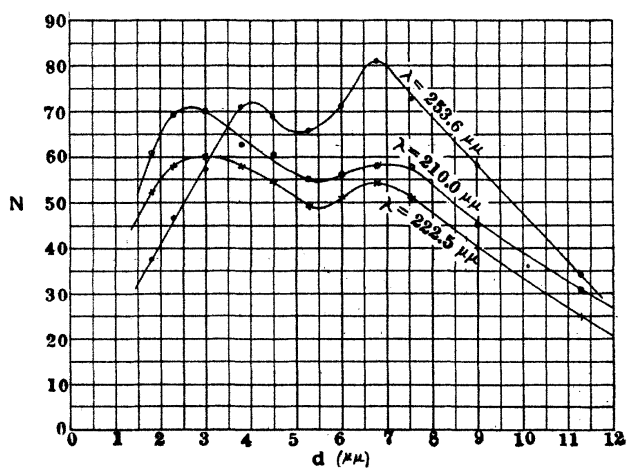

Fig. 9.

but there are two points of difference. The second maximum is relatively smaller and the first maximum is displaced toward the left when dealing with the shorter wave-lengths. Every set of films tested showed these characteristics, although they were more pronounced in some sets than in others.

(c) Time Changes.-An examination of Fig. 8 shows that even for a given wave-length, the two maxima are not always of the same relative magnitude. This is due to a slow time change in the properties of the films, an actual example of which is shown by Fig. Io. The second maximum becomes relatively less important as the film stands, and it makes no apparent difference whether the films stand in vacuo, or exposed to air. Heating the films in an oven at about $150^{\circ} \mathrm{C}$., however, greatly accelerates the change, and after this treatment the second hump is scarcely noticable.

\footnotetext{
${ }^{1}$ King, Phys. Rev., I0, p. 291, 191 7.
} 
Discussion of Results. Interpretation of the Two Maxima.-Apparently the two maxima of the curves must be ascribed to platinum in two conditions. Of these conditions, the one giving rise to the right hand maximum is evidently more unstable and more electropositive than the other, since it is relatively more important soon after the formation of the films and relatively more sensitive to light of long wave-lengths. We therefore take the first maximum to be characteristic of platinum in its ordinary stable state, while the second maximum is due

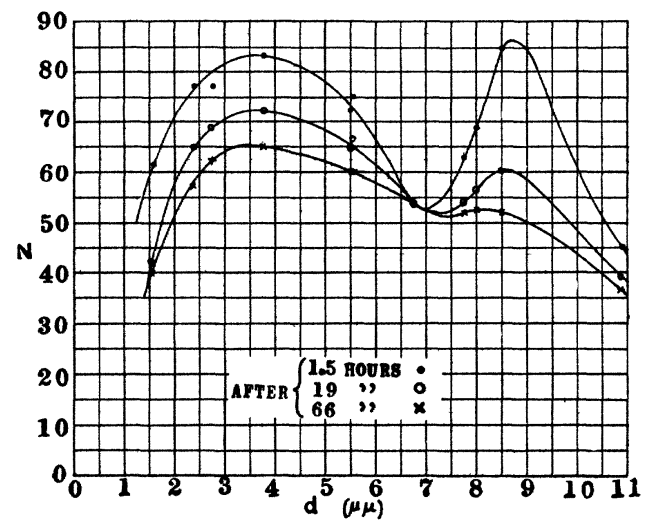

Fig. 10.

to platinum in the amorphous or spongy state in which it is first deposited. Since the change from the amorphous to the stable state is spontaneous, the latter state must be one of less internal potential energy, which is in accord with the observation that the platinum in this state is more electronegative. It is also seen, by reference to Figs. 2 and 4 , that the average distance, $I / \beta$ or $I / \gamma$, moved by an electron without losing its ability to escape is greater in the less compact state. These deductions are all consistent with each other and are also in line with well known facts regarding the abnormal resistivity of freshly sputtered films and the change to normal resistivity produced by "aging." We shall therefore confine the remaining discussion to the information regarding ordinary platinum which is given by a study of the first maximum.

Comparison of the Three Assumptions Regarding the Process by which an Electron Loses its Ability to Escape.-The third assumption, viz., that an escaping electron loses energy in direct proportion to the distance traveled through the metal, may at once be discarded. According to it, the thickness giving maximum emission should be directly proportional to $\left(\nu-\nu_{0}\right)$ by equation $\mathrm{I} 2$, or to $\left(\mathrm{I} / \lambda-\mathrm{I} / \lambda_{0}\right)$, where $\lambda$ is the wave-length of the exciting light and $\lambda_{0}$ is the longest wave-length of light which will 
cause photoelectric emission. $\lambda_{0}$ is known to be about $280 \mu \mu$ for platinum. ${ }^{1}$ The values of $\lambda$ employed ranged from $210 \mu \mu$ to $265 \mu \mu$. Thus the assumption requires that $d_{m}$ should be four or five times larger for the shorter than for the longer wave-lengths. Not only is this not the case, but what little variation of $d_{m}$ with $\lambda$ is found is in the opposite direction to that required by the assumption.

As far as we can see, either the first or the second assumptions are in conformity with the facts. We are unable to decide between them experimentally, since either equation (2) or equation (5) satisfies the experimental results within the limits of uncertainty introduced by the presence of the second maximum. Of the two assumptions, however, the more reasonable one is the second-viz., that the probability that an electron will retain its ability to escape decreases exponentially with the distance traveled from its starting point.

On this assumption, we may determine $\mathrm{I} / \gamma$, the average distance which an electron may move without losing its ability to escape. For $\lambda=253.6 \mu \mu$, we may take $d_{m}=3.75 \mu \mu$ as the mean of all our determinations. Taking $\alpha=.078 \mu \mu^{-1}$, we find $\gamma$, from Fig. 4 , to be $0.375 \mu \mu^{-1}$.

For shorter wave-lengths, $d_{m}$ has slightly smaller values. This may be due to a larger value of $\alpha$ for shorter wave-lengths or to a larger value of $\gamma$. This latter possibility is very unlikely, since it would mean that the rapidly moving electrons have less average penetrating power than the slow ones. We attempted measurements of $\alpha$ for the shorter wavelengths and did succeed in proving that $\alpha$ increases as $\lambda$ is decreased below about $245 \mu \mu$, but our measurements were not accurate enough to prove that the shift of $d_{m}$ with wave-length is due entirely to this cause. We are, however, safe in saying that there is no evidence that $I / \gamma$ increases as $\lambda$ decreases, $i$. e., there is no evidence that electrons with large initial velocities retain their ability to escape over longer paths than the slower electrons.

This result is rather surprising and is of considerable importance, for it proves that an electron loses its ability to escape at a single catastrophe, and not by gradual process or a succession of small energy losses. If we define one of these catastrophes as a "collision," then we may identify $\mathrm{I} / \gamma$ with the "mean free path" of the electron in the metal. This "mean free path" is apparently independent of the velocity of the electron, within the limits of velocity of photoelectrons. It is not easy to see how the theory of free electrons moving like molecules of a perfect gas in a metal can be reconciled with these results which indicate definite collisions of an inelastic type. These experiments are therefore consistent 
with the facts of specific heats of metals in indicating that electrons cannot exist in metals freely and independently sharing the thermal energy with the atoms.

The magnitude of the mean free path $\mathrm{I} / \gamma$ is 2.67 (I0) ${ }^{-7} \mathrm{~cm}$., which is greater than, but of the same order of magnitude as the distance between atomic centers in platinum. It is therefore probable that the electrons lose at least the greater part of their kinetic energy at encounters with atoms, and they may entirely lose their freedom at these encounters.

Patterson ${ }^{1}$ has calculated the mean free path of conducting electrons by data regarding the Hall effect in platinum and also by applying $\mathrm{J}$. $\mathrm{J}$. Thomson's theory of the resistance of thin films. By each method he finds the mean free path to be about $6(\mathrm{Io})^{-7} \mathrm{~cm}$., not far different from the value we have calculated. Strict comparison is inadmissible, however, because the "mean free path" does not mean quite the same thing in the two cases, and because the assumptions underlying the formulæ used by Patterson cannot be accepted in their present form.

Gold FILMs. In dealing with gold films the experimental difficulties were so great as to preclude the possibility of any great accuracy, and no extensive tests were made. The difficulties arise from the high resistence of films of the thickness necessary for the present tests, and are apparently due to lack of continuity of the films. Isolated portions of the films quickly charge up to retarding potentials, causing the rate of electrometer deflection to decrease rapidly. This phenomenon was noticed with only the very thinnest films of platinum.

In order to be more independent of errors from this cause, the films were kept shielded from light until the electrometer key was opened and we were ready to make an observation. A shutter was opened, so as to expose the film for two seconds. The deflection due to the electrons liberated in this interval was taken to represent the relative photoelectric sensitiveness of the film. The results of these tests are shown in Fig. II. There is no evidence of a double maximum, though the determinations may not be accurate enough to make this conclusion certain. The mean free path $\mathrm{I} / \gamma$ may be calculated by Fig. 4 to be about 5.0 (I0) ${ }^{-7} \mathrm{~cm}$. The value of $\alpha$ used in this calculation is given by Meier ${ }^{2}$ as $\alpha=.056 \mu \mu^{-1}$ for wave-length $253.6 \mu \mu$. Patterson has suggested a value 16 (I0) ${ }^{-7} \mathrm{~cm}$. for the mean free path of the conducting electrons, while Partzsch and Hallwachs suggest

$$
\frac{\mathrm{I}}{\beta}=\mathrm{II} .6(\mathrm{IO})^{-7} \mathrm{~cm} .
$$

(See the second assumption of the present paper.)

1 Phil. Mag., 3, p. 655, 1902.

${ }^{2}$ Loc. cit. 
Summary.-The experiments described in this paper indicate that:

I. Photoelectrons, excited within a metal, lose their initial kinetic energy as the result of single and definite catastrophes, or "collisions," rather than by a gradual process or a succession of small energy losses.

2. The average distance which an electron can move before it loses its

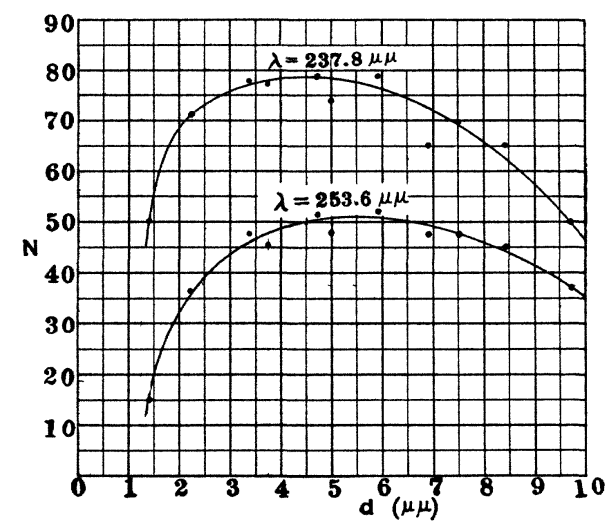

Fig. 11.

energy by one of these "collisions" is independent of the initial velocity and is of the same order as, but slightly larger than, the distance between atoms. The measurements indicate a mean free path of about 2.67 (I0) $)^{-7} \mathrm{~cm}$. in platinum and $5.0(\mathrm{IO})^{-7} \mathrm{~cm}$. in gold.

3. Platinum films thinner than $2 \mu \mu$ and gold films thinner than about $8 \mu \mu$ are apt to be imperfectly conducting due to isolated regions which are not in conducting contact with the main body of the film. Platinum films therefore appear to be more homogeneous than gold films.

4. The small penetrating power of photo-electrons renders it extremely improbable that the thermionic emission from metals may be accounted for by considering it to be a photoelectric effect from the entire body of the metal integrated over the complete radiation spectrum. ${ }^{1}$

${ }^{1}$ This possibility is discussed in detail in Richardson's "The Emission of Electricity from Hot Bodies," pp. 95-102.

2 Since the above paper was written, a paper dealing with the same general subject has been published in the February number of this Journal by Dr. Otto Stuhlman, Jr. His experimental results are not in good agreement with those in this paper, and his interpretation of them is quite different. We are at present unable to suggest a satisfactory explanation of the apparent lack of agreement.

Palmer Physical laboratory,

PRINCETON, N. J. 


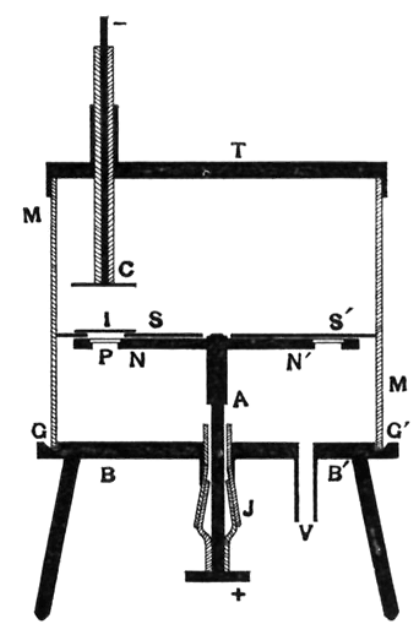

Fig. 5. 


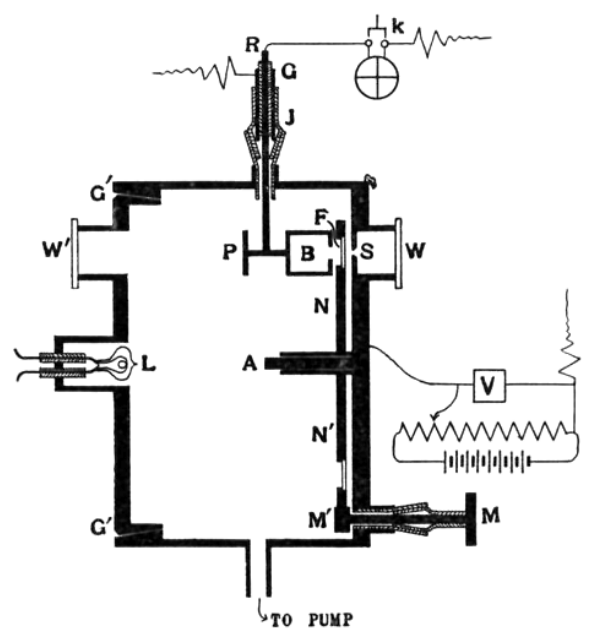

Fig. 7. 